Original Research Paper

\title{
Application of Homotopy Perturbation Method for SIR Model with Vital Dynamics and Constant Population
}

\author{
Mohammed S. Mechee and Ghassan A. Al-Juaifri \\ Department of Mathematics, Faculty of Computer Science and Mathematics, Kufa University, Najaf, Iraq
}

Article history

Received: $24-07-2017$

Revised: $19-08-2017$

Accepted: 03-01-2018

Corresponding Author:

Mohammed S. Mechee

Department of Mathematics,

Faculty of Computer Science

and Mathematics, Kufa

University, Najaf, Iraq

Email: mohammeds.abed@uokufa.edu.iq

\begin{abstract}
In this work, we have studied Susceptible-Infected-Recovered (SIR) model with vital dynamics and constant population, which is used as a mathematical models in many physically significant fields of applied science. The Homotopy Perturbation Method (HPM) and Runge-Kutta method (RK) have been used for solving the SIR model with vital dynamics and constant population. The convergence of HPM has been studied. Also, we have tested the HPM on solving different implementations which are show the efficiency and accuracy of the method. The approximated solutions of HPM for the tested problems are agree well with the numerical solutions of Runge-Kutta method.
\end{abstract}

Keyword: Susceptible-Infected-Recovered Model, Homotopy, HPM, Partial Differential Equation, System

\section{Introduction}

The most important mathematical models for physical phenomena is the differential equation. Motion of objects, Fluid and heat flow, bending and cracking of materials, vibrations, chemical reactions and nuclear reactions are all modeled by systems of Differential Equations (DEs). Moreover, Numerous mathematical models in science and engineering are expressed in terms of unknown quantities and their derivatives. Many applications of DEs, particularly Ordinary Differential Equations (ODEs) of different orders, can be found in the mathematical modeling of real life problems (Mechee et al., 2014).

The Homotopy Perturbation Method (HPM), which are a well-known, is efficient technique to find the approximate solutions for ordinary and Partial Differential Equations (PDEs) which describe different fields of science, physical phenomena, engineering, mechanics and so on. HPM was proposed by He (1999) for solving linear and nonlinear Des and Integral Equations (IEs). HPM has many advantages for solving numerous classes of scientific and engineering experiments which are lead to ODEs, PDEs and IEs. HPM gives the approximated solutions of these problems as a series. The approximated solutions using this method mostly are convergent. It is worth to note that the major advantage of He's homotopy perturbation method is that the perturbation equation can be freely constructed in many ways and approximation can also be freely selected. Many researchers used HPM to approximate the solutions of DEs and IEs (Yildirim, 2010; Jalaal et al., 2010; Ma et al., 2008). Many researchers published some papers on solving some classes DEs using HPM. For example, Chun and Sakthivel (2010) used HPM for solving a linear and nonlinear secondorder two-point boundary value problems while Gülkaç (2010) solved the Black-Scholes equation for a simple European option in this method to obtain a new efficient recurrent relation to solve BlackScholes equation. Moreover, numerous researches used HPM for solving nonlinear DEs, Vahidi et al. (2011) solved nonlinear DEs, which yields the Maclaurin series of the exact solution, Chang and Liou (2006) developed a third-order explicit approximation to find the roots of the dispersion relation for water waves that propagate over dissipative media, Zhou and $\mathrm{Wu}$ (2012) solved the nonlinear PB equation describing spherical and planar colloidal particles immersed in an arbitrary valence and mixed electrolyte solution, Özis and Akç (2011) solved certain non-linear, non-smooth oscillators, Yazdi (2013) solved nonlinear vibration analysis of functionally graded plate while He and Huan (2004) applied HPM for solving nonlinear oscillators with discontinuities, nonlinear Duffing equation and some nonlinear ODEs. For class of linear PDEs, Al-Saif and Abood (2011) solved the Korteweg-de Vries (KdV) equation and convergence study of HPM, Babolian et al. (2009) used HPM to solve time-dependent DEs, Aswhad and Jaddoa (2016) solved advection Problem, vibrating beam equation linear and nonlinear PDEs and the system of nonlinear PDEs and Babolian et al. (2009) used the homotopy perturbation method to solve time-dependent differential. Also, many researchers used HPM for solving the class of non-linear PDEs, Yazdi (2013) provided approximated 
solution for free nonlinear vibration of thin rectangular laminated FGM plates, Liao (2004) solved nonlinear PDEs, Yildirim (2009) was used to implement the nonlinear Korteweg-de Vries equation. Taghipour (2010) solved parabolic equations and Periodic equation linear and nonlinear PDEs. Janalizadeh et al. (2008) obtained the solution of a second-order non-linear wave equation, Fereidoon et al. (2011) utilized to derive approximate explicit analytical solution for the nonlinear foam drainage equation, Momani and Odibat (2007) modified the algorithm which provides approximate solutions in the form of convergent series with easily computable components, Babolian et al. (2009) solved time-dependent DEs while He (2000), solved non-linear problems using the homotopy technique. However, for the system of differential equations, Bataineh et al. (2009) solved systems of secondorder Boundary Value Problems (BVPs), Javidi (2009) solved SIR model, Wang and Song (2007) studied the solution of a human Immune Virus Model (HIV) infection of $C D 4^{+} T$ cells which are also called as leukocytes or $T$ helper cells, Rafei et al. (2007) obtained the solution of the system of nonlinear ODEs governing on the problem, Noor et al. (2013) solved the system of linear equations. Noor and Mohyud-Din (2008) obtained the solution of linear and non-linear sixth-order boundary value problems and system of differential equations. Also, Javidi (2009) solved system of Linear Fredholm Integral Equations (LFIEs). Yusufoglu (2009) solved a linear Fredholm type integro-differential equations with separable kernel. Javidi (2009) solved non-linear Fredholm IEs, Saberi-Nadja and Tamamgar (2008) used modified HPM for solving the system linear and nonlinear of Volterra integral equations, Kumar et al. (2011) solved generalized Abel integral equation. Lastly, for the DEs of fractional type, Odibat and Momani (2008) solved nonlinear DEs of fractional order, Jafari et al. (2010) solved nonlinear problems of fractional Riccati differential equation and Yildirim and Agirseven (2009) solved the space-time fractional advection-dispersion equation. Recently, we have studied the general SIR model which is a system of DEs which are used as a mathematical model in physically significant field of applied science. We have introduced the homotopy perturbation method for solving generalized SIR model. Also, we have tested the HPM on the solving of different implementations which are show the efficiency and accuracy of the proposed method. The approximated solutions are agree wellwith analytical solutions for the tested problems. It has been highlighted that the use of HPM is more suitable to approximate the solutions of DEs with considering the general coefficient functions.

\section{Preliminary}

\section{Homotopy Perturbation Method (HPM)}

In this section, we present a brief description of the HPM, to illustrate the basic ideas of the HPM, we consider the following DE (Neamaty and Darzi, 2010; Chun and Sakthivel, 2010; Batiha, 2015; Abbasbandy, 2006):

$A(u)-f(\tau)=0, \quad \tau \in \Omega$

with boundary conditions:

$$
B\left(u, \frac{\partial u}{\partial \tau}\right)=0, \quad \tau \in \partial \Omega
$$

where, $t$ is independent variable, $u$ is dependent variable, $A$ is general differential operator, $B$ is a boundary operator, $f(t)$ is a known analytic function and $\partial \Omega$ is the boundary of the domain $\Omega$. The operator $A$ can be generally divied into two parts of $L$ and $N$ where $L$ is linear part, while $N$ is the nonlinear part in the DE, Therefore Equation (1) can be rewritten as follows (He, 1999):

$$
L(u)+N(u)-f(\tau)=0
$$

By using homotopy technique, one can construct a homotopy:

$$
V(\tau, p): \Omega \times[0,1] \mapsto R
$$

which satisfies:

$$
H(v, p)=(1-p)\left[L(v)-L\left(u_{0}\right)\right]+p[L(v)+N(v)-f(\tau)]=0
$$

or:

$$
H(v, p)=L(v)-L\left(u_{0}\right)+p L\left(u_{0}+p[N(v)-f(\tau)]\right)=0
$$

where, $p \in[0,1], \tau \in \Omega$ and $p$ is called homotopy parameter, $H$ is homotopy function and $u_{o}$ is an initial approximation for the solution of Equation (1) which satisfies the boundary conditions obviously. Using Equation (4) or (5), we have the following equation:

$$
H(v, 0)=L(v)-L\left(u_{0}\right)=0
$$

and:

$$
H(v, 1)=L(v)+N(v)-f(\tau)=0
$$

Assume that the solution of (4) or (5) can be expressed as a series in $p$ as follows:

$$
V=v_{0}+p v_{1}+p^{2} v_{2}+p^{3} v_{3}+\cdots=\sum_{i=0}^{\infty} p^{i} v_{i}
$$

set $p \rightarrow 1$ in the approximated solution of (1). 
Table 1: List of dependent variables and parameters of the SIR model

\begin{tabular}{ll}
\hline $\begin{array}{l}\text { Dependent variables } \\
\text { and parameters }\end{array}$ & Meaning \\
\hline Dependent variables & Susceptibles \\
$S(t)$ & Infectives \\
$L(t)$ & Recovered with immunity \\
$R(t)$ & \\
Parameters & Average death rate \\
$\mu$ & Contact rate \\
$\beta$ & Average infectious period \\
$\frac{1}{\lambda}$ & \\
\hline
\end{tabular}

Consequently:

$$
u(\tau)=\lim _{p \rightarrow 1} V=v_{0}+v_{2}+v_{3}+\cdots=\sum_{i=0}^{\infty} v_{i}
$$

\section{Susceptible-Infected-Recovered Model (SIR)}

We will consider the following system of SIR model of ODEs:

$\frac{d S(t)}{d t}=\mu-\mu S(t)-\beta L(t) S(t)$

$\frac{d L(t)}{d t}=\beta L(t) S(t)-(\lambda+\mu) L(t)$,

$\frac{d R(t)}{d t}=\lambda L(t)-\mu R(t)$,

with the initial conditions:

$S(0)=a 1, L(0)=a 2, R(0)=a 3$.

where, $a 1, a 2$ and $a 3$ are given constants. The dependent variables and the parameters of the SIR model are given in Table 1.

A general technique for solving the SIR model with vital dynamics and constant population is described in the following algorithms: (Beretta and Takeuchi, 1997; Shulgin et al., 1998).

\section{Analysis of HPM for Solving the SIR Model with Vital Dynamics and Constant Population}

In this section, we will present a review of the HPM for solving SIR model with vital dynamics and constant population.

\section{Algorithm of Solving SIR Model}

Firstly, we start with the initial approximations (13).

Secondly, we can construct a homotopy for DEs system (10)-(12) as follows:

$$
\begin{aligned}
& H(S(t), p)=(1-p)\left(\frac{d S(t)}{d t}-\frac{d S_{0}(t)}{d t}\right) \\
& +p\left(\frac{d S(t)}{d t}-\mu+\mu S(t)+\beta L(t) S(t)\right)=0 \\
& H(L(t), p)=(1-p)\left(\frac{d L(t)}{d t}-\frac{d L_{0}(t)}{d t}\right) \\
& +p\left(\frac{d L(t)}{d t}-\beta L(t) S(t)+\lambda L(t)+\mu L(t)\right)=0 \\
& H(R(t), p)=(1-p)\left(\frac{d R(t)}{d t}-\frac{d R_{0}(t)}{d t}\right) \\
& +p\left(\frac{d R(t)}{d t}-\lambda L(t)+\mu R(t)\right)=0
\end{aligned}
$$

Thirdly, suppose that the solutions of the Equations (14-16) are in the following forms:

$$
\begin{aligned}
& S(t)=S_{0}(t)+p S_{1}(t)+p^{2} S_{2}(t)+p^{3} S_{3}(t)+\ldots \\
& L(t)=L_{0}(t)+p L_{1}(t)+p^{2} L_{2}(t)+p^{3} L_{3}(t)+\ldots \\
& R(t)=R_{0}(t)+p R_{1}(t)+p^{2} R_{2}(t)+p^{3} R_{3}(t)+\ldots
\end{aligned}
$$

Therefore:

$$
\begin{aligned}
& H(S(t), p)=(1-p)\left(\sum_{i=0}^{\infty} p^{i} \frac{d S_{i}(t)}{d t}-\frac{d S_{0}(t)}{d t}\right) \\
& +p\left(\begin{array}{l}
\sum_{i=0}^{\infty} p^{i} \frac{d S_{i}(t)}{d t}-\mu+\sum_{i=0}^{\infty} p^{i} \mu S_{i}(t) \\
+\beta \sum_{i=0}^{\infty} p^{i} L_{i}(t) \sum_{i=0}^{\infty} p^{i} S_{i}(t)
\end{array}\right)=0 \\
& H(L(t), p)=(1-p)\left(\sum_{i=0}^{\infty} p^{i} \frac{d L_{i}(t)}{d t}-\frac{d L_{0}(t)}{d t}\right) \\
& +p\left(\begin{array}{l}
\sum_{i=0}^{\infty} p^{i} \frac{d L_{i}(t)}{d t}-\beta \sum_{i=0}^{\infty} p^{i} L_{i}(t)+\sum_{i=0}^{\infty} p^{i} S_{i}(t) \\
+\sum_{i=0}^{\infty} p^{i} \lambda L_{i}(t)+\sum_{i=0}^{\infty} p^{i} \mu L_{i}(t)
\end{array}\right)=0 \\
& H(R(t), p)=(1-p)\left(\sum_{i=0}^{\infty} p^{i} \frac{d R_{i}(t)}{d t}-\frac{d R_{0}(t)}{d t}\right) \\
& +p\left(\sum_{i=0}^{\infty} p^{i} \frac{d R_{i}(t)}{d t}-\sum_{i=0}^{\infty} p^{i} \lambda L_{i}(t)+\sum_{i=0}^{\infty} p^{i} \mu R_{i}(t)\right)=0
\end{aligned}
$$

Fourthly, by collecting terms of the same power of $p$ we obtain the following equations:

$p^{0}: \frac{d S_{0}(t)}{d t}-\frac{d S_{0}(t)}{d t}=0$ 


$$
\begin{aligned}
& \frac{d L_{0}(t)}{d t}-\frac{d L_{0}(t)}{d t}=0 \\
& \frac{d R_{0}(t)}{d t}-\frac{d R_{0}(t)}{d t}=0 \\
& p^{1}: \frac{d S_{1}(t)}{d t}+\frac{d S_{0}(t)}{d t}-\mu+\left(\mu+\beta L_{0}(t)\right) S_{0}(t)=0 \\
& \frac{d L_{1}(t)}{d t}+\frac{d L_{0}(t)}{d t}+\left(\lambda-\beta S_{0}(t)+\mu\right) L_{0}(t)=0 \\
& \frac{d R_{1}(t)}{d t}+\frac{d R_{0}(t)}{d t}-\lambda L_{0}(t)+\mu R_{0}(t)=0 \\
& p^{2}: \frac{d S_{2}(t)}{d t}+\mu S_{1}(t)+\beta\left(L_{0}(t) S_{1}(t)+L_{1}(t) S_{0}(t)\right)=0 \\
& \frac{d L_{2}(t)}{d t}+(\lambda+\mu) L_{1}(t)-\beta\left(L_{0}(t) S_{1}(t)+L_{1}(t) S_{0}(t)\right)=0 \\
& \frac{d R_{2}(t)}{d t}-\lambda L_{1}(t)+\mu R_{1}(t)=0
\end{aligned}
$$

Hence, in general for $m=3,4,5, \ldots$ we have:

$$
\begin{aligned}
& p^{m}: \frac{d S_{m}(t)}{d t}+\mu S_{m-1}(t)+\beta\left(\sum_{j=0}^{m-1} L_{j}(t) S_{n-j-1}(t)\right)=0 \\
& \frac{d L_{m}(t)}{d t}+(\lambda+\mu) L_{m-1}(t)-\beta\left(\sum_{j=0}^{m-1} L_{j}(t) S_{n-j-1}(t)\right)=0 \\
& \frac{d R_{m}(t)}{d t}-\lambda L_{m-1}(t)+\mu R_{m-1}(t)=0
\end{aligned}
$$

Finally, by using the Equations (23-34) with some simplifications, we get the following sequence of the solutions:

$$
\begin{aligned}
& S_{0}(t)=a 1, L_{0}(t)=a 2, R_{0}(t)=a 3 \\
& S_{1}(t)=-\int\left(\frac{d S_{0}(t)}{d t}-\mu+\left(\mu+\beta L_{0}(t)\right) S_{0}(t)\right) d t \\
& =(-a 1 a 2 \beta-a 1 \mu+\mu) t \\
& L_{1}(t)=-\int\left(\frac{d L_{0}(t)}{d t}+\left(\lambda-\beta S_{0}(t)+\mu\right) L_{0}(t)\right) d t \\
& =a 2(a 1 \beta-\lambda-\mu) t
\end{aligned}
$$

$$
\begin{aligned}
& R_{1}(t)=-\int\left(\frac{d R_{0}(t)}{d t}-\lambda L_{0}(t)+\mu R_{0}(t)\right) d t=(a 2 \lambda-a 3 \mu) t \\
& S_{2}(t)=-\int\left(\mu S_{1}(t)+\beta\left(L_{0}(t) S_{1}(t)+L_{1}(t) S_{0}(t)\right)\right) d t \\
& =\frac{1}{2}\left(\begin{array}{l}
a 1^{2} a 2 \beta^{2}+a 1 a 2^{2} \beta^{2}-a 1 a 2 \beta \lambda \\
+a 1 a 2 \beta \mu+a 1 \mu^{2}-\mu a 2 \beta-\mu^{2}
\end{array}\right) t^{2} \\
& \begin{array}{l}
L_{2}(t)=-\int\left(\begin{array}{l}
(\lambda+\mu) L_{1}(t) \\
-\beta\left(L_{0}(t) S_{1}(t)+L_{1}(t) S_{0}(t)\right)
\end{array}\right) d t \\
=\frac{1}{2} a 2 t^{2}\left(\begin{array}{l}
a 1^{2} \beta^{2}-a 1 a 2 \beta^{2}-2 a 1 \beta \lambda-3 a 1 \beta \mu \\
+\beta \mu+\lambda^{2}+2 \lambda \mu+\mu^{2}
\end{array}\right) \\
R_{2}(t)=-\int\left(-\lambda L_{1}(t)+\mu R_{1}(t)\right) d t \\
=\frac{1}{2}\left(a 1 a 2 \beta \lambda-\lambda^{2} a 2-2 \lambda \mu a 2+a 3 \mu^{2}\right) t^{2}
\end{array}
\end{aligned}
$$

$$
\begin{aligned}
& S_{3}(t)=-\int\left(\mu S_{2}(t)+\beta\left(\begin{array}{l}
L_{0}(t) S_{2}(t) \\
+L_{1}(t) S_{1}(t)+L_{0}(t) S_{2}(t)
\end{array}\right)\right) d t \\
& =\left(\begin{array}{l}
-\frac{1}{6} a 1^{3} a 2 \beta^{3}+\frac{1}{3} \beta^{3} a 2^{2} a 1^{2}-\frac{1}{6} \beta^{3} a 2^{3} a 1 \\
+\frac{1}{3} a 1^{2} a 2 \beta^{2} \lambda+\frac{2}{3} \mu a 1^{2} a 2 \beta^{2}-\frac{1}{6} \beta^{2} a 2^{2} a 1 \lambda \\
-\frac{2}{3} \mu a 1 a 2^{2} \beta^{2}-\frac{1}{2} a 1 a 2 \beta^{2} \mu-\frac{1}{6} a 1 a 2 \beta \lambda^{2} \\
-\frac{1}{2} \mu a 1 a 2 \beta \lambda-\frac{5}{6} \mu^{2} a 1 a 2 \beta+\frac{1}{6} \beta^{2} a 2^{2} \mu \\
-\frac{1}{6} \mu^{3} a 1+1 / 3 a 2 \beta \lambda \mu+\frac{2}{3} \mu^{2} a 2 \beta+\frac{1}{6} \mu^{3}
\end{array}\right) t^{3}
\end{aligned}
$$$$
L_{3}(t)=-\int\left(\begin{array}{l}
(\lambda+\mu) L_{2}(t)- \\
\beta\left(L_{0}(t) S_{2}(t)+L_{1}(t) S_{1}(t)+L_{0}(t) S_{2}(t)\right)
\end{array}\right) d t
$$$$
=\frac{1}{6} a 2 t^{3}\left(\begin{array}{l}
a 1^{3} \beta^{3}-2 a 1^{2} a 2 \beta^{3}+a 1 a 2^{2} \beta^{3}-3 a 1^{2} \beta^{2} \lambda \\
-6 a 1^{2} \beta^{2} \mu+2 a 1 a 2 \beta^{2} \lambda+4 a 1 a 2 \beta^{2} \mu+3 a 1 \beta^{2} \mu \\
+3 a 1 \beta \lambda^{2}+9 a 1 \beta \lambda \mu+7 a 1 \beta \mu^{2}-a 2 \beta^{2} \mu \\
-3 \beta \lambda \mu-4 \beta \mu^{2}-\lambda^{3}-3 \lambda^{2} \mu-3 \lambda \mu^{2}-\mu^{3}
\end{array}\right)
$$

$$
\begin{aligned}
& R_{3}(t)=-\int\left(-\lambda L_{2}(t)+\mu R_{2}(t)\right) d t \\
& =\left(\begin{array}{l}
\frac{1}{6} a 1^{2} a 2 \beta^{2} \lambda-\frac{1}{6} \beta^{2} a 2^{2} a 1 \lambda- \\
\frac{1}{3} a 1 a 2 \beta \lambda^{2}-\frac{2}{3} \mu a 1 a 2 \beta \lambda+\frac{1}{6} a 2 \beta \lambda \mu+\frac{1}{6} \lambda^{3} a 2 \\
+\frac{1}{2} \lambda^{2} \mu a 2+\frac{1}{2} \lambda \mu^{2} a 2-\frac{1}{6} \mu^{3} a 3
\end{array}\right) t^{3}
\end{aligned}
$$




$$
\begin{aligned}
S_{4}(t) & =-\int\left(\mu S_{3}(t)+\beta\left(L_{0}(t) S_{3}(t)+L_{1}(t) S_{2}(t)+L_{2}(t) S_{1}(t)+L_{3}(t) S_{0}(t)\right)\right) d t \\
& =\left(\begin{array}{l}
\frac{1}{40} a 1^{4} \beta^{5} a 2^{2}-\frac{1}{40} a 1^{3} \beta^{5} a 2^{3}+\frac{1}{40} a 1^{4} a 2 \beta^{4} \mu-\frac{1}{20} a 1^{3} a 2^{2} \beta^{4} \lambda \\
-\frac{1}{10} a 1^{3} a 2^{2} \beta^{4} \mu-\frac{1}{40} a 1^{3} a 2 \beta^{4} \mu-\frac{1}{20} a 1^{3} a 2 \beta^{3} \lambda \mu-\frac{3}{40} a 1^{3} a 2 \beta^{3} \mu^{2} \\
+\frac{1}{20} a 1^{2} a 2^{2} \beta^{4} \mu+\frac{1}{40} a 1^{2} \beta^{3} a 2^{2} \lambda^{2}+\frac{1}{20} a 1^{2} a 2^{2} \beta^{3} \lambda \mu+\frac{1}{40} a 1^{2} \beta^{3} a 2^{2} \mu^{2} \\
+\frac{1}{20} a 1^{2} a 2 \beta^{3} \lambda \mu+\frac{1}{10} a 1^{2} a 2 \beta^{3} \mu^{2}+\frac{1}{40} a 1^{2} a 2 \beta^{2} \lambda^{2} \mu+\frac{1}{20} a 1^{2} a 2 \beta^{3} \lambda \mu^{2} \\
+\frac{1}{40} a 1^{2} a 2 \beta^{2} \mu^{3}-\frac{1}{40} a 1 a 2 \beta^{3} \mu^{2}-\frac{1}{40} a 1 a 2 \beta^{2} \mu^{2}-\frac{1}{20} a 1 a 2 \beta^{2} \lambda \mu^{2} \\
-\frac{1}{40} a 1 a 2 \beta^{2} \mu^{3}
\end{array}\right) t^{5} \\
& +\left(\begin{array}{l}
\frac{5}{6} a 1 a 2 \beta^{2} \mu^{2}-\frac{1}{6} a 1^{2} a 2 \beta^{3} \mu+\frac{1}{24} a 1 \mu^{4}-\frac{1}{24} \mu^{4} \\
-\frac{5}{8} \mu a 1^{2} a 2 \beta^{2} \lambda+\frac{5}{12} \mu a 2^{2} a 1 \beta^{2} \lambda+\frac{7}{24} \mu a 1 a 2 \beta \lambda^{2}+\frac{5}{8} \mu^{2} a 2 a 1 \beta \lambda \\
+\frac{7}{24} a 1 a 2 \beta^{2} \lambda \mu-\frac{1}{3} \mu^{2} \beta^{2} a 2^{2}-\frac{11}{24} \mu^{3} a 2 \beta+\frac{1}{24} \beta^{4} a 2^{2} a 1^{3}-\frac{3}{8} \beta^{4} a 2^{3} a 1^{2} \\
+\frac{1}{24} \beta^{4} a 2^{4} a 1-\frac{1}{24} \beta^{3} a 2^{3} \mu-\frac{1}{24} \beta^{4} a 2 a 1^{4}-\frac{1}{8} a 2 \beta^{2} \mu-\frac{1}{8} a 2 \beta \lambda^{2} \mu \\
+\frac{1}{8} a 1^{3} \beta^{3} a 2 \lambda-\frac{1}{8} a 1^{2} \beta^{2} a 2 \lambda^{2}+\frac{1}{24} a 1 \beta a 2 \lambda^{3}+\frac{1}{3} \mu a 1^{3} a 2 \beta^{3}-\frac{5}{6}, \mu \beta^{3} a 2^{2} a 1^{2} \\
+\frac{1}{3} \mu \beta^{3} a 2^{3} a 1-\frac{7}{8} \mu^{2} a 1^{2} a 2 \beta^{2}+\frac{5}{8} \mu^{2} a 1 a 2^{2} \beta^{2}+\frac{13}{24} \mu \mu^{3} a 1 a 2 \beta-\frac{11}{24} \mu^{2} a 2 \beta \lambda \\
-\frac{1}{12} \beta^{3} a 2^{2} a 1^{2} \lambda+\frac{1}{6} \beta^{3} a 2^{3} a 1 \lambda+\frac{13}{24} \beta^{3} a 2^{2} a 1 \mu+\frac{1}{24} \beta^{2} a 2^{2} a 1 \lambda^{2}-\frac{5}{24} \beta^{2} a 2^{2} \lambda \mu
\end{array}\right) t^{4}
\end{aligned}
$$

$$
\left.\begin{array}{rl}
L_{4}(t)=-\int\left((\lambda+\mu) L_{3}(t)-\beta\left(L_{0}(t) S_{3}(t)+L_{1}(t) S_{2}(t)+L_{2}(t) S_{1}(t)\right)+L_{3}(t) S_{0}(t)\right) d t \\
=-\frac{1}{24} a 2\left(\begin{array}{l}
245^{2} \\
a 1 \\
a
\end{array} \beta^{3} \lambda \mu-\frac{9}{5} a 1 a 2 \beta^{2} \lambda^{2} \mu-\frac{9}{5} a 1 a 2 \beta^{2} \lambda \mu^{2}-3 a 1^{3} a 2 \beta^{4} \mu\right. \\
+\frac{3}{5} a 1^{2} a 2^{2} \beta^{4} \mu+3 a 1^{2} a 2 \beta^{3} \mu^{2}-\frac{6}{5} a 1 a 2 \beta^{3} \mu^{2}-\frac{3}{5} a 1 a 2 \beta^{2} \mu^{3}+\frac{9}{5} a 1^{2} \beta^{3} \lambda \mu \\
-\frac{9}{5} a 1^{3} a 2 \beta^{4} \lambda+\frac{3}{5} a 1^{2} a 2^{2} \beta^{4} \lambda-\frac{9}{5} a 1^{3} \beta^{3} \lambda \mu+\frac{6}{5} a 1^{2} a 2 \beta^{4} \mu+\frac{9}{5} a 1^{2} a 2 \beta^{3} \lambda^{2} \\
+\frac{9}{5} a 1^{2} \beta^{2} \lambda^{2} \mu+\frac{21}{5} a 1^{2} \beta^{2} \lambda \mu^{2}-\frac{3}{5} a 1 a 2 \beta^{2} \lambda^{3}-\frac{9}{5} a 1 \beta^{2} \lambda^{2} \mu-\frac{24}{5} a 1 \beta^{2} \lambda \mu^{2} \\
-\frac{3}{5} a 1 \beta \lambda^{3} \mu-\frac{9}{5} a 1 \beta \lambda^{2} \mu^{2}-\frac{9}{5} a 1 \beta \lambda \mu^{3}+\frac{3}{5} \beta \mu^{4}+\frac{3}{5} \beta^{2} \mu^{3}-\frac{6}{5} a 1 a 2 \beta^{3} \lambda \mu \\
+3 a 1^{2} \beta^{3} \mu^{2}-\frac{12}{5} a 1^{3} \beta^{3} \mu^{2}+\frac{12}{5} a 1^{2} \beta^{2} \mu^{3}-\frac{3}{5} a 1^{3} \beta^{5} a 2^{2}+\frac{3}{5} a 1^{4} a 2 \beta^{5}+\frac{3}{5} a 1^{4} \beta^{4} \mu \\
-\frac{3}{5} a 1^{3} \beta^{4} \mu-\frac{3}{5} a 1 \beta^{3} \mu^{2}-3 a 1 \beta^{2} \mu^{3}-\frac{3}{5} a 1 \beta \mu^{4}+\frac{3}{5} \beta^{2} \lambda \mu^{2}+\frac{3}{5} \beta \lambda^{3} \mu+\frac{9}{5} \beta \lambda^{2} \mu^{2}+\frac{9}{5} \beta \lambda \mu^{3}
\end{array}\right) t^{5}
$$




$$
\begin{aligned}
R_{4}(t)= & -\int\left(-\lambda L_{3}(t)+\mu R_{3}(t)\right) d t \\
& =\left(\begin{array}{l}
-\frac{1}{40} a 1^{3} a 2^{2} \beta^{4} \lambda+\frac{1}{40} a 1^{2} a 2^{3} \beta^{4} \lambda-\frac{1}{40} a 1^{3} a 2 \beta^{3} \lambda \mu+\frac{1}{20} a 1^{2} \beta^{3} a 2^{2} \lambda^{2} \\
+\frac{1}{10} a 1^{2} a 2^{2} \beta^{3} \lambda \mu+\frac{1}{40} a 1^{2} a 2 \beta^{3} \lambda \mu+\frac{1}{20} a 1^{2} a 2 \beta^{2} \lambda^{2} \mu+\frac{3}{40} a 1^{2} a 2 \beta^{2} \lambda \mu^{2} \\
-\frac{1}{20} a 1 a 2^{2} \beta^{3} \lambda \mu-\frac{1}{40} a 1 a 2^{2} \beta^{2} \lambda^{3}-\frac{1}{20} a 1 a 2^{2} \beta^{2} \lambda^{2} \mu-\frac{1}{40} a 1 a 2^{2} \beta^{2} \lambda \mu^{2} \\
-\frac{1}{20} a 1 a 2 \beta^{2} \lambda^{2} \mu-\frac{1}{10} a 1 a 2 \beta^{2} \lambda \mu^{2}-\frac{1}{40} a 1 a 2 \beta \lambda^{3} \mu-\frac{1}{40} a 1 a 2 \beta \lambda^{2} \mu^{2} \\
-\frac{1}{40} a 1 a 2 \beta \lambda \mu^{3}+\frac{1}{40} a 2 \beta^{2} \lambda \mu^{2}+\frac{1}{40} a 2 \beta \lambda^{3} \mu+\frac{1}{40} a 2 \beta \lambda^{2} \mu^{2}+\frac{1}{40} a 2 \beta \lambda \mu^{3}
\end{array}\right) t^{5} \\
& +\left(\begin{array}{l}
\frac{1}{24} a 1^{3} \beta^{3} a 2 \lambda+\frac{1}{24} \beta^{3} a 2^{3} a 1 \lambda-\frac{1}{8} a 1^{2} \beta^{2} a 2 \lambda^{2}-\frac{5}{24} \mu a 1^{2} a 2 \beta^{2} \lambda \\
+\frac{1}{8} \mu \beta^{2} a 2^{2} a 1 \lambda+\frac{1}{24} a 1 a 2 \beta^{2} \lambda \mu+\frac{1}{8} a 1 \beta a 2 \lambda^{3}+\frac{3}{8} \mu a 1 a 2 \beta \lambda^{2}+\frac{3}{8} \mu^{2} a 1 a 2 \beta \lambda \\
-\frac{1}{24} \beta^{2} a 2^{2} \lambda \mu-\frac{1}{24} a 2 \beta \lambda^{2} \mu-\frac{1}{8} \mu^{2} a 2 \beta \lambda-\frac{1}{24} \lambda^{4} a 2-\frac{1}{6}, \lambda^{3} \mu a 2-\frac{1}{4} \lambda^{2} \mu^{2} a 2 \\
+\frac{1}{6} \lambda \mu^{3} a 2+\frac{1}{24} \mu^{4} a 3
\end{array}\right) t^{4}
\end{aligned}
$$

Hence, the solutions of the system (10-12) with the initial conditions (13) as follows:

$$
\begin{aligned}
& S(t)=S_{0}(t)+S_{1}(t)+S_{2}(t)+S_{3}(t)+S_{4}(t)+\ldots \\
& L(t)=L_{0}(t)+L_{1}(t)+L_{2}(t)+L_{3}(t)+L_{4}(t)+\ldots
\end{aligned}
$$

and:

$$
R(t)=R_{0}(t)+R_{1}(t)+R_{2}(t)+R_{3}(t)+R_{4}(t)+\ldots
$$

\section{Convergence of the Method}

In this section, we have explain the convergence of HPM for SIR model

\section{Definition 3.1}

Convergence of the series.

Let $u_{1}(x), u_{2}(x), u_{3}(x), \ldots, u_{n}(x), \ldots$ be a sequence of functions. The series $\sum_{n=1}^{\infty} u_{n}(x)$ is said to be converge to the function $u(x)$ if the sequence $\left\{s_{n}(x)\right\}_{n=0}^{\infty}$ of partial sums defined by $s_{n}(x)=\sum_{k=1}^{n} u_{k}(x)$ converge to $u(x)$.

HPM is convergent method if the series in (9) is convergent. The sufficient condition for convergence of the HPM for the systems of nonlinear ordinary differential equations is the series $\sum_{n=1}^{\infty} u_{n}(x)$ to be converge to $u(x)$. From initial conditions (35), we have bounded functions $S_{0}(t), L_{0}(t)$ and $R_{0}(t)$ on the domain of $t \in\left[0, t_{0}\right]$. Consequently, from (36), (37) and (38), we can conclude the following:

$$
\begin{aligned}
\left|S_{1}(t)\right| & \leq \int\left|\left(\frac{d S_{0}(t)}{d t}-\mu+\left(\mu+\beta L_{0}(t)\right) S_{0}(t)\right)\right| d t \\
& \leq|(-a 1 a 2 \beta-a 1 \mu+\mu) t| \\
& \leq \alpha 1
\end{aligned}
$$

$$
\begin{aligned}
\left|L_{1}(t)\right| & \leq \int\left|\left(\frac{d L_{0}(t)}{d t}+\left(\lambda-\beta S_{0}(t)+\mu\right) L_{0}(t)\right)\right| d t \\
& \leq|a 2(a 1 \beta-\lambda-\mu) t|, \\
& \leq \beta_{1}
\end{aligned}
$$

and:

$$
\begin{aligned}
R_{1}(t) & \leq \int\left|\left(\frac{d R_{0}(t)}{d t}-\lambda L_{0}(t)+\mu R_{0}(t)\right)\right| d t \\
& \leq|(a 2 \lambda-a 3 \mu) t|, \\
& \leq \gamma_{1}
\end{aligned}
$$

Hence, The functions $S_{1}(t), L_{1}(t)$ and $R_{1}(t)$ are bounded in the domain of $t$.

Consequently:

$$
\begin{aligned}
& \left|S_{2}(t)\right| \leq \int\left|\left(\mu S_{1}(t)+\beta\left(L_{0}(t) S_{1}(t)+L_{1}(t) S_{0}(t)\right)\right)\right| d t \\
& \leq \frac{1}{2}\left|\left(\begin{array}{l}
a 1^{2} a 2 \beta^{2}+a 1 a 2^{2} \beta^{2}-a 1 a 2 \beta \lambda \\
+a 1 a 2 \beta \mu+a 1 \mu^{2}-\mu a 2 \beta-\mu^{2}
\end{array}\right) t^{2}\right| \\
& \leq \alpha^{2}
\end{aligned}
$$




$$
\begin{aligned}
& \left|L_{2}(t)\right| \leq \int\left|\left((\lambda+\mu) L_{1}(t)-\beta\left(L_{0}(t) S_{1}(t)+L_{1}(t) S_{0}(t)\right)\right)\right| d t \\
& \leq\left|\frac{1}{2} a 2 t^{2}\left(\begin{array}{l}
a 1^{2} \beta^{2}-a 1 a 2 \beta^{2}-2 a 1 \beta \lambda \\
-3 a 1 \beta \mu+\beta \mu+\lambda^{2}+2 \lambda \mu+\mu^{2}
\end{array}\right)\right| \\
& \leq \beta_{2}
\end{aligned}
$$

and:

$$
\begin{aligned}
\left|R_{2}(t)\right| & \leq \int\left|\left(-\lambda L_{1}(t)+\mu R_{1}(t)\right)\right| d t \\
& \leq \frac{1}{2}\left|\left(a 1 a 2 \beta \lambda-\lambda^{2} a 2-2 \lambda \mu a 2+a 3 \mu^{2}\right) t^{2}\right| \\
& \leq \gamma_{2}
\end{aligned}
$$

However, the functions $S_{2}(t), L_{2}(t)$ and $R_{2}(t)$ are bounded in the domain of $t$, where $\alpha_{i}, \beta_{i}$ and $\gamma_{i}$ are constant for $i=1,2, \ldots$.

In general, for $n=3,4, \ldots$, the the functions $S_{n}(t), L_{n}(t)$ and $R_{n}(t)$ are bounded in the domain of $t$ where domain of $t$ is $\left[0, t_{0}\right]$.

\section{Theorem 3.1 (Biazar and Ghazvini, 2009)}

Suppose that $X$ and $Y$ be Banach space and $N: X \rightarrow Y$ is a contraction nonlinear mapping, that is:

$$
\forall v, \tilde{v} \in X ;\|N(v)-N(\tilde{v})\| \leq \gamma\|v-\tilde{v}\|, 0<\gamma<1
$$

Which according to Banach's fixed point theorem, having fixed point fixed point $u$, that is $N(u)=u$. The sequence generated by HPM will be regarded as:

$$
V_{n}=N\left(V_{n-1}\right), V_{n-1}=\sum_{i=0}^{n-1} u_{i}, n=1,2,3, \ldots
$$

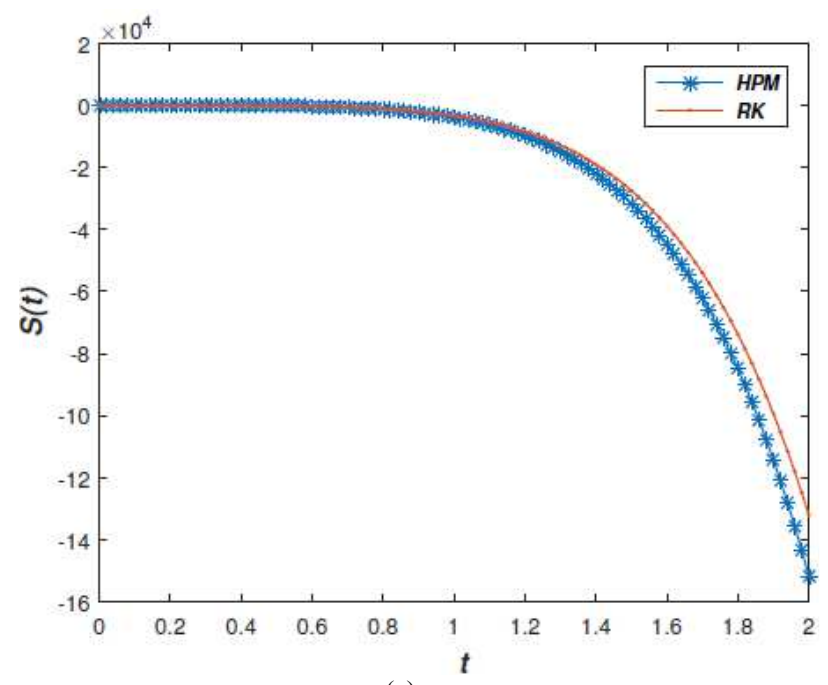

(a) and suppose that $V_{0}=v_{0}=u_{0} \in B_{r}(u)$ where $B_{r}(u)=\left\{u^{*} \in X\left\|u^{*}-u\right\|<r\right\}$, then we have the following statement:

1. $\left\|V_{n}-u\right\| \leq \gamma^{n}\left\|v_{0}-u\right\|$

2. $V_{n} \in B_{r}(u)$

3. $\lim _{n \rightarrow \infty} V_{n}=u$

Proof

By the induction method on $n$.

Using Theorem 3.1, the series (48), (49) and (50) are convergent to $S(t), L(t)$ and $R(t)$ respectively. Then, we have:

$$
\begin{aligned}
& \lim _{n \rightarrow \infty} S_{n}(t)=S(t) \\
& \lim _{n \rightarrow \infty} L_{n}(t)=L(t)
\end{aligned}
$$

and:

$$
\lim _{n \rightarrow \infty} R_{n}(t)=R(t)
$$

\section{Implementations}

In order to assess the accuracy of solving SIR model with vital dynamics and constant population using HPM we will introduce some models in different parameters and the logistic growth of the healthy $C D^{4} T$ cells to examine the dynamics of a model for HIV infection of $C D^{4} T$ cells. The results of the following three problems for numerical comparison between the present method and classical Runge-Kutta method of order 4 (Faires and Burden, 2003), which mentioned in the literature has been presented through and Fig. 1 to 3.

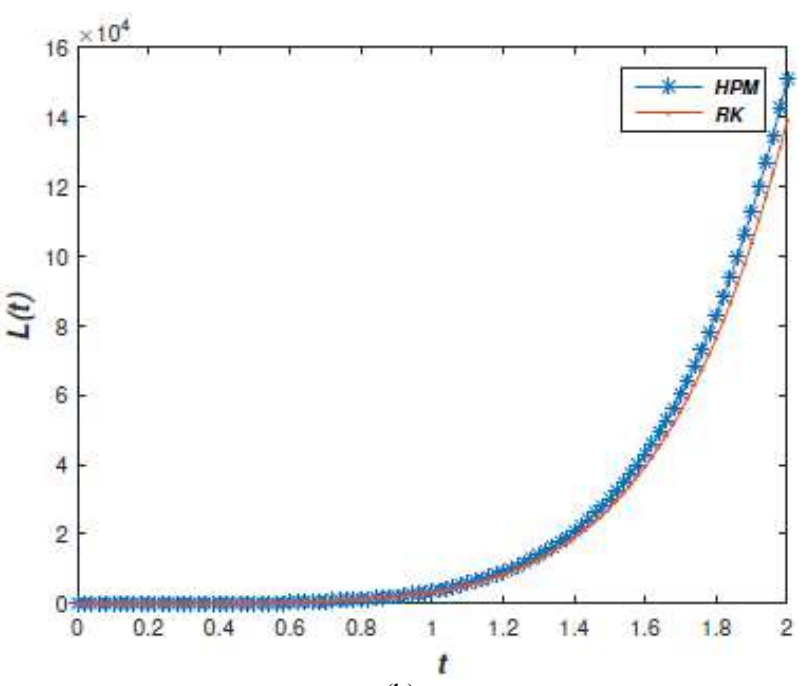

(b) 


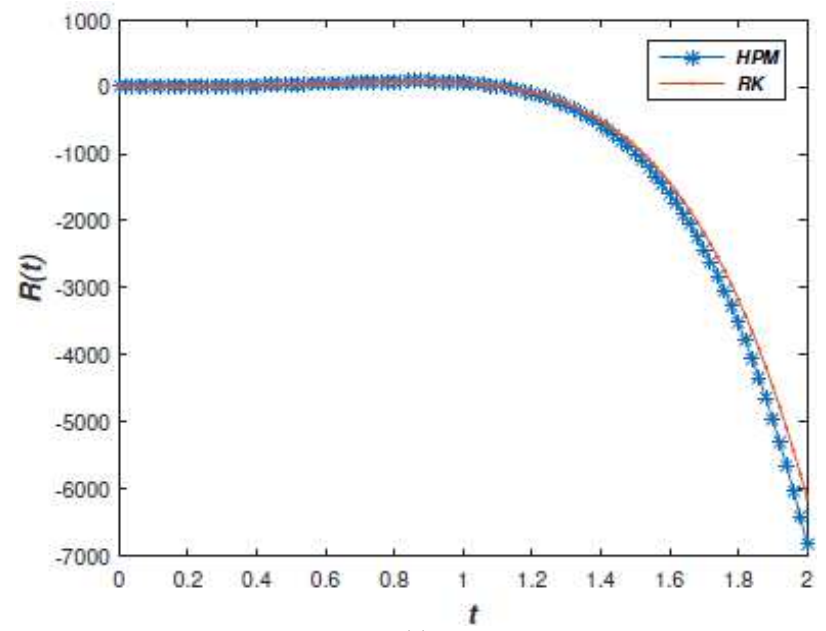

(c)

Fig. 1: Comparisons on (a) $S(t)$, (b) $L(t)$ and (c) $R(t)$ resp. versus $t$ for Problem 1 using homotopy perturbation method and RungeKutta method with the initial conditions $S_{0}(t)=20, L_{0}(t)=5, R_{0}(t)=5$ and the parameters $\mu=0.16, \beta=0.5, \lambda=1.5$ and $t_{0}=2$

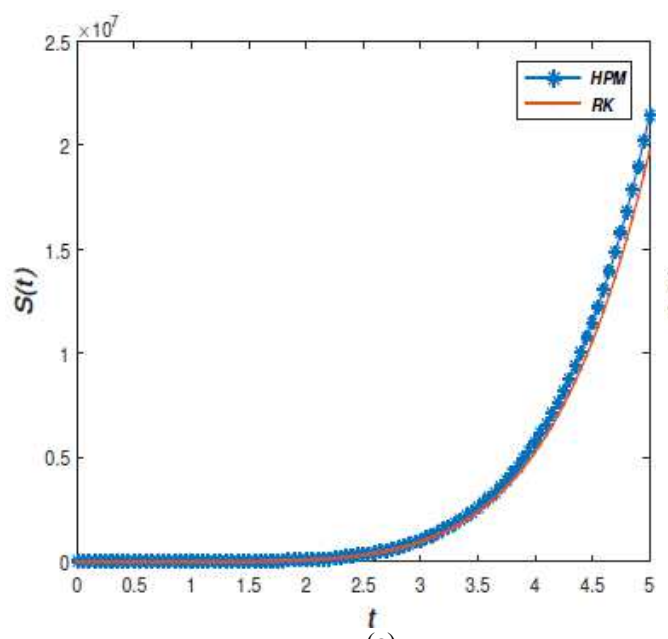

(a)

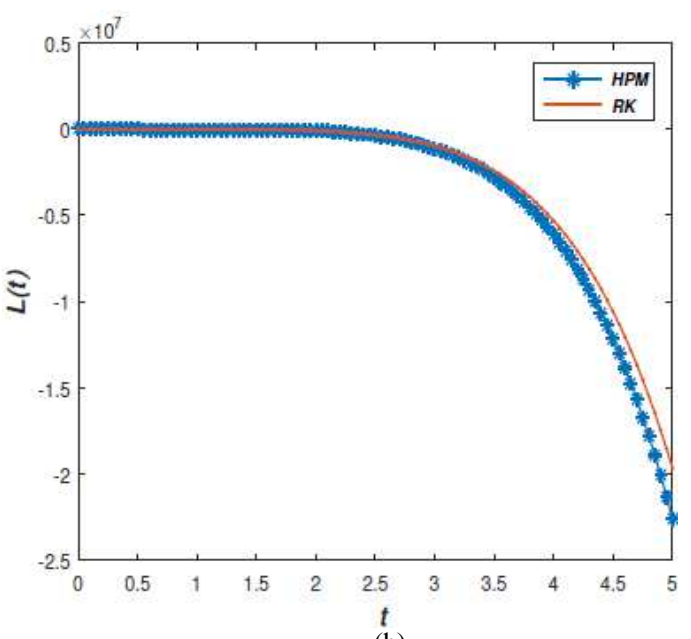

(b)

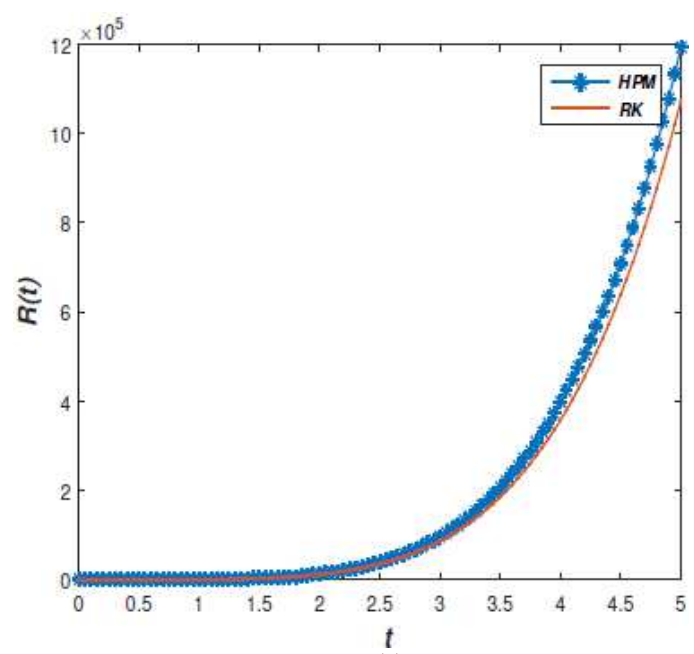

(c)

Fig. 2: Comparisons on (a) $S(t)$, (b) $L(t)$ and (c) $R(t)$ resp. versus $t$ for Problem 2 using homotopy perturbation method and RungeKutta method with the initial conditions $S_{0}(t)=15, L_{0}(t)=10, R_{0}(t)=5$ and the parameters $\mu=1, \beta=0.3, \lambda=2,4$ and $t_{0}=5$ 


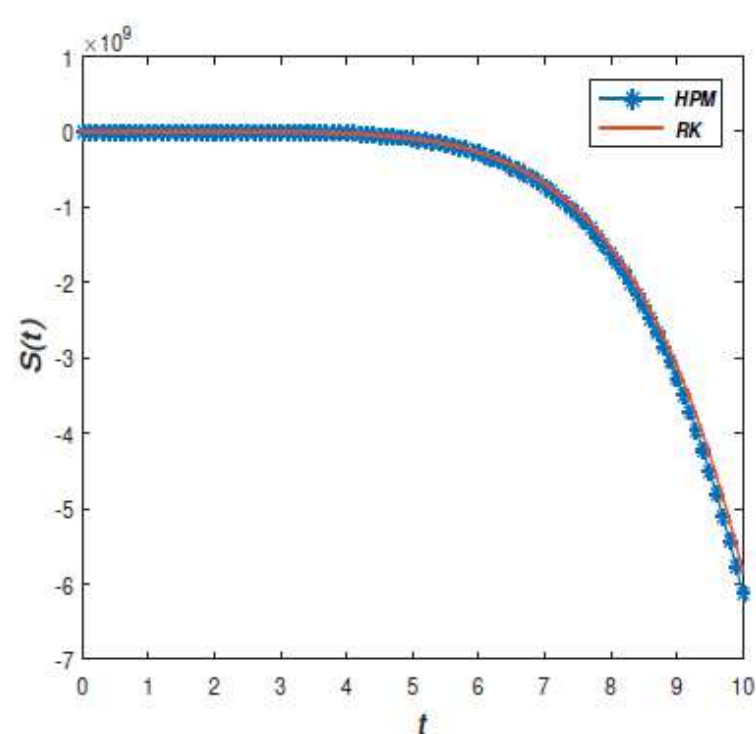

(a)

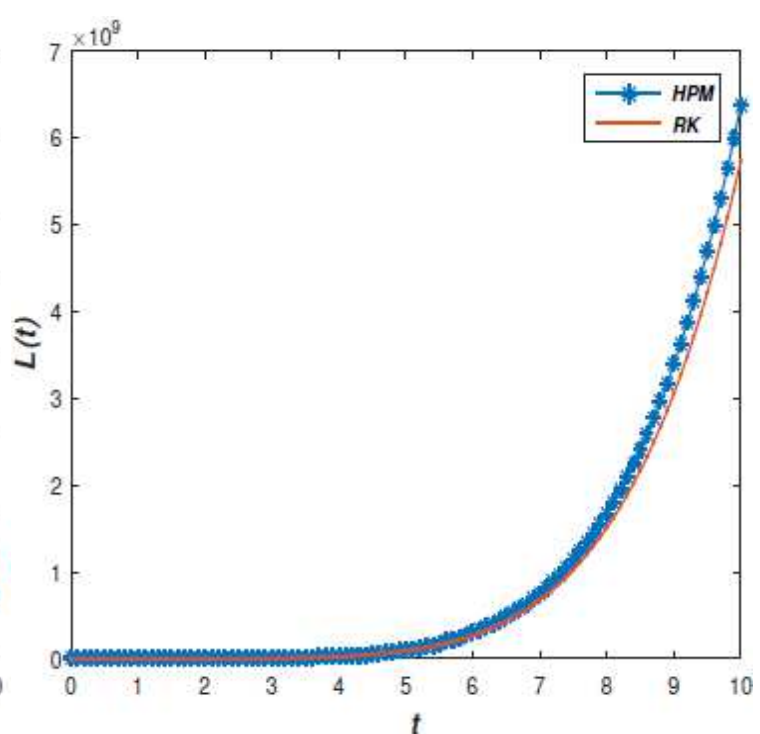

(b)

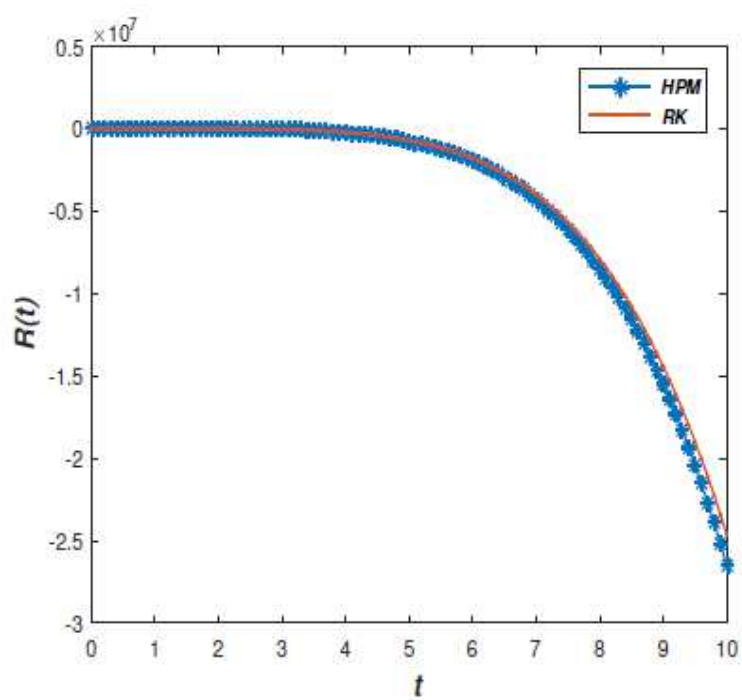

(c)

Fig. 3: Comparisons on (a) $S(t)$, (b) $L(t)$ and (c) $R(t)$ resp. versus $t$ for Problem 3 using homotopy perturbation method and RungeKutta method with the initial conditions $S_{0}(\mathrm{t})=10, L_{0}(t)=3, R_{0}(t)=1$ and the parameters $\mu=0.072, \beta=1.2, \lambda=0: 5$ and $t_{0}$ $=10$

\section{Problem 1}

Consider the following initial conditions and parameters of SIR model, $S_{0}(t)=20, L_{0}(t)=5, R_{0}(t)=5$, $\mu=0.16, \beta=0.5$ and $\lambda=1.5$.

Substituting these parameters in the Equations (48), (49) and (50), we have the general solutions of the system (10-12) which are written as follows:

$$
\begin{aligned}
& S(t)=-1802.309410 t^{6}-150.8775971 t^{5} \\
& -1912.396658 t^{4}-237.4203039 t^{3} \\
& +279.0432000 t^{2}-53.04 t+20
\end{aligned}
$$

$$
\begin{aligned}
& L(t)=2093.446578 t^{6}-115.925287 t^{5} \\
& +987.9505310 t^{4}+531.6334200 t^{3}+ \\
& 107.5890000 t^{2}+41.70 t+5 \\
& R(t)=-427.9890420 t^{5}+394.5526581 t^{4} \\
& +52.15508667 t^{3}+30.73900000 t^{2}+6.70 t+5
\end{aligned}
$$

\section{Problem 2}

Consider the following initial conditions and parameters of SIR model, $S_{0}(t)=15, L_{0}(t)=10, R_{0}(t)=5$, $\mu=1, \beta=0.3$ and $\lambda=2,4$. 
Substituting these parameters in the Equations (48), (49) and (50), we have the general solution of system $(10-12)$ which is written as follows:

$$
\begin{aligned}
& S(t)=1234.987630 t^{6}+802.2399453 t^{5} \\
& -607.4347917 t^{4}-1.758333333 t^{3} \\
& +142.7500000 t^{2}-59.0 t+15 \\
& L(t)=-1225.744986 t^{6}-1277.164016 t^{5} \\
& +877.0750417 t^{4}+112.5183334 t^{3} \\
& -82.45000000 t^{2}+11.0 t+10 \\
& R(t)=350.2476000 t^{5}+168.6186667 t^{4} \\
& -67.19333333 t^{3}+3.700000000 t^{2}+19.0 t+5
\end{aligned}
$$

\section{Problem 3}

Consider the following initial conditions and parameters of SIR model, $S_{0}(t)=10, L_{0}(t)=3, R_{0}(t)=1$, $\mu=0.072, \beta=1.2$ and $\lambda=0.5$

Substituting these parameters in the Equations (48), (49) and (50), we have the general solution of system $(10-12)$ which is written as follows:

$$
\begin{aligned}
& S(t)=-6038.675734 t^{6}-562.2567630 t^{5} \\
& -3524.368751 t^{4}-351.2929183 t^{3} \\
& +272.9897280 t^{2}-36.648 \mathrm{t}+10 \\
& L(t)=6308.801712 t^{6}+385.976322 t^{5} \\
& +1984.539802 t^{4}+822.5434045 t^{3} \\
& +129.9323760 t^{2}+34.284 t+3 \\
& R(t)=-285.7057029 t^{5} \\
& +204.8636178 t^{4}+21.45092579 t^{3} \\
& +8.519592000 t^{2}+1.428 t+1
\end{aligned}
$$

\section{Discussion and Conclusion}

In this study, a class of the SIR model with vital dynamics and constant population has been introduced. HPM has been used for solving the SIR model. The approximated solutions of implementations have been derived using Maple. A Comparison of the results which are obtained by homotopy perturbation method with Runge-Kutta method, using MATLAB, shows that the approximated solutions of HPM are agree well with numerical solutions of RK method for the tested problems Moreover, the approximated solutions are efficient and high accurate.

\section{Acknowledgement}

The authors would like to thank university of Kufa for supporting this research project.

\section{Author's Contributions}

Both authors equally contributed in modeling, analysis, simulation and writing up this work.

\section{Ethics}

This article is original and contains unpublished material. The corresponding author confirms that all of the other authors have read and approved the manuscript and there are no ethical issues involved.

\section{References}

Abbasbandy, S., 2006. Iterated he's homotopy perturbation method for quadratic riccati differential equation. Applied Math. Comput., 175: 581-589. DOI: 10.1016/j.amc.2005.07.035

Al-Saif, A. and D.A. Abood, 2011. The homotopy perturbation method for solving $\mathrm{K}(2,2)$ equation. J. Basrah Res. Sci., 37: 151-157.

Aswhad, A.A. and A.F. Jaddoa, 2016. The approximate solution of newell whitehead segel and fisher equations using the adomian decomposition method. Al-Mustansiriyah J. Sci., 25: 4556-4556

Babolian, E., A. Azizi and J. Saeidian, 2009. Some notes on using the homotopy perturbation method for solving time-dependent differential equations. Math. Comp. Mod., 50: 213-224. DOI: 10.1016/j.mcm.2009.03.003

Bataineh, A.S., M.S.M. Noorani and I. Hashim, 2009. Modified homotopy analysis method for solving systems of second-order BVPs. Commun. Nonlinear Sci. Numerical Simulat., 14: 430-442. DOI: 10.1016/j.cnsns.2007.09.012

Batiha, B., 2015. A new efficient method for solving quadratic Riccati differential equation. Int. J. Applied Math. Res., 4: 24-29. DOI: 10.14419/ijamr.v4il.4113

Beretta, E. and Y. Takeuchi, 1997. Convergence results in SIR epidemic models with varying population sizes. Nonlinear Anal. Theory Meth. Applic., 28: 1909-1921. DOI: 10.1016/S0362-546X(96)00035-1

Biazar, J. and H. Ghazvini, 2009. Convergence of the homotopy perturbation method for partial differential equations. Nonlinear Anal. Real World Applic., 10: 2633-2640. DOI: 10.1016/j.nonrwa.2008.07.002

Chang, H.K. and J.C. Liou, 2006. Solving wave dispersion equation for dissipative media using homotopy perturbation technique. J. Waterway Port. Coastal, Ocean Eng., 132: 28-35. 
Chun, C. and R. Sakthivel, 2010. Homotopy perturbation technique for solving two-point boundary value problems-comparison with other methods. Comput. Phys. Commun., 181: 1021-1024 DOI: $10.1016 /$ j.cpc.2010.02.007

Faires, J.D. and R. Burden, 2003. Numerical methods. Thomson Learning, Inc., Pacific Grove.

Fereidoon, A., H. Yaghoobi and M. Davoudabadi, 2011. Application of the homotopy perturbation method for solving the foam drainage equation. Int. J. Diff. Eq., 2011: 864023-864023. DOI: 10.1155/2011/864023

Gülkaç, V., 2010. The homotopy perturbation method for the Black-Scholes equation. J. Stat. Comput. Simulat., 80: 1349-1354. DOI: 10.1080/00949650903074603

He, J. and Huan, 2004. The homotopy perturbation method for nonlinear oscillators with discontinuities. Applied Math. Comput., 151: 287-292. DOI: 10.1016/S0096-3003(03)00341-2

He, J.H., 1999. Homotopy perturbation technique. Comput. Meth. Applied Mechan. Eng., 178: 257-262. DOI: $10.1016 / \mathrm{S} 0045-7825(99) 00018-3$

He, J.H., 2000. A coupling method of a homotopy technique and a perturbation technique for non-linear problems. Int. J. Non-linear Mechan., 35: 37-43. DOI: $10.1016 / \mathrm{S} 0020-7462(98) 00085-7$

Jafari, H., N. Kadkhoda, H. Tajadodi and S.H. Matikolai, 2010. Homotopy perturbation pade technique for solving fractional Riccati differential equations. Int. J. Nonlinear Sci. Numer. Simul., 11: 271-275.

Jalaal, M., D. Ganji and F. Mohammadi, 2010. He's homotopy perturbation method for twodimensional heat conduction equation: Comparison with fnite element method. Heat Transfer-Asian Res., 39: 232-245. DOI: 10.1002/htj.20292

Janalizadeh, A., A. Barari and D. Ganji, 2008. IOP Publishing, 012080.

Javidi, M., 2009. Modified homotopy perturbation method for solving system of linear Fredholm integral equations. Math. Comput. Modell., 50: 159-165. DOI: $10.1016 /$ j.mcm.2009.02.003

Kumar, S., O.P. Singh and S. Dixit, 2011. Generalized abel inversion using homotopy perturbation method. Applied Math., 2: 254-257.

DOI: $10.4236 / \mathrm{am} .2011 .22029$

Liao, S., 2004. On the homotopy analysis method for nonlinear problems. Applied Math. Comput., 147: 499-513. DOI: 10.1016/S0096-3003(02)00790-7

Ma, X., L. Wei and Z. Guo, 2008. He's homotopy perturbation method to periodic solutions of nonlinear Jerk equations, J. Sound Vibrat., 314: 217-227. DOI: 10.1016/j.jsv.2008.01.033

Mechee, M., F. Ismail, Z. Hussain and Z. Siri, 2014. Direct numerical methods for solving a class of third-order partial differential equations. Applied Math. Comput., 247: 663-674.

DOI: $10.1016 /$ j.amc.2014.09.021
Momani, S. and Z. Odibat, 2007. Homotopy perturbation method for nonlinear partial differential equations of fractional order. Phys. Lett. A, 365: 345-350. DOI: 10.1016/j.physleta.2007.01.046

Neamaty, A. and R. Darzi, 2010. Comparison between the variational iteration method and the homotopy perturbation method for the Sturm-Liouville differential equation. Boundary Value Prob., 2010: 317-369. DOI: 10.1155/2010/317369

Noor, M.A. and S.T. Mohyud-Din, 2008. Homotopy perturbation method for solving sixth-order boundary value problems. Comput. Math. Applic., 55: 2953-2972. DOI: 10.1016/j.camwa.2007.11.026

Noor, M.A., K.I. Noor, S. Khan and M. Waseem, 2013. Modified homotopy perturbation method for solving system of linear equations. J. Assoc. Arab Uni. Basic Applied Sci., 13: 35-37. DOI: $10.1016 /$ j.jaubas.2012.07.004

Odibat, Z. and S. Momani, 2008. Modified homotopy perturbation method: Application to quadratic Riccati differential equation of fractional order. Chaos, Solitons Fractals, 36: 167-174. DOI: 10.1016/j.chaos.2006.06.041

Özis, T. and C. Akç, 2011. Periodic solutions for certain non-smooth oscillators by iterated homotopy perturbation method combined with Modified Lindstedt-Poincaré technique. Meccanica, 46: 341-347. DOI: 10.1007/s11012-010-9312-1

Rafei, M., D. Ganji and H. Daniali, 2007. Solution of the epidemic model by homotopy perturbation method. Applied Math. Comput., 187: 1056-1062. DOI: $10.1016 /$ j.amc.2006.09.019

Saberi-Nadja, J. and M. Tamamgar, 2008. Modified homotopy perturbation method for solving the system of Volterra integral equations. Int. J. Nonlinear Sci. Numerical Simulat., 9: 409-41.

Shulgin, B., L. Stone and Z. Agur, 1998. Pulse vaccination strategy in the SIR epidemic model. Bull. Math. Biol., 60: 1123-1148. DOI: 10.1006/S0092-8240(98)90005-2

Taghipour, R., 2010. Application of homotopy perturbation method on some linear and nonlinear periodic equations. World Applied Sci. J., 10: 1232-1235.

Vahidi, A., E. Babolian and Z. Azimzadeh, 2011. An improvement to the homotopy perturbation method for solving nonlinear Duffing's equations. Bull. Malaysian Math. Sci. Society.

Wang, X. and X. Song, 2007. Global stability and periodic solution of a model for HIV infection of $C D 4^{+} T$ cells. Applied Math. Comput., 189: 1331-1340. DOI: 10.1016/j.amc.2006.12.044

Yazdi, A.A., 2013. Homotopy perturbation method for nonlinear vibration analysis of functionally graded plate. J. Vibrat. Acoust., 135: 021012-021012. DOI: $10.1115 / 1.4023252$ 
Yildirim, A. and D. Agirseven, 2009. The homotopy perturbation method for solving singular initial value problems. Methods, 3: 5-5.

DOI: $10.1515 /$ IJNSNS.2009.10.2.235

Yildirim, A., 2009. On the solution of the nonlinear Korteweg-de Vries equation by the homotopy perturbation method. Int. J. Numerical Meth. Biom. Eng., 25: 1127-1136. DOI: 10.1002/cnm.1146

Yildirim, A., 2010. He's homotopy perturbation method for nonlinear differential-difference equations. Int. J. Comput. Math., 87: 992-996.

DOI: $10.1080 / 00207160802247646$
Yusufoglu, E., 2009. Improved homotopy perturbation method for solving Fredholm type integrodifferential equations. Chaos Solitons Fractals, 41: 28-37. DOI: 10.1016/j.chaos.2007.11.005

Zhou, S. and H. Wu, 2012. Analytical solutions of nonlinear Poisson-Boltzmann equation for colloidal particles immersed in a general electrolyte solution by homotopy perturbation technique. Colloid Polymer Sci., 290: 1165-1180. 